\title{
Was bleibt nach zehn Jahren Olympische Jugendspiele?
}

\author{
Martin Schnitzer, Thomas Schwarzbauer
}

\section{Abstract}

Im Jahr 2007 wurde mit den Olympischen Jugendspiele (YOG) ein neues olympisches Event ins Leben gerufen. Die Spiele werden alle zwei Jahre abwechselnd als Winter- und Sommersportevent ausgetragen. Ziel ist es, vor allem junge Menschen zum Sport zu motivieren und ihnen die olympischen Werte näher zu bringen sowie neue Innovationen und Konzepte auszuprobieren. Die Entstehung sowie die Durchführung der YOG wurde wissenschaftlich begleitet. Dabei wurden verschiedenste Themen untersucht, und interessante Punkte konnten aufgezeigt werden:

- Die YOG bieten für Athletinnen und Athleten eine unvergessliche Erfahrung, die oft als Karrierehighlight gesehen wird.

- Die Teilnahme an den YOG kann ist kein Prädiktor für den weiteren Karriereverlauf.

- Das Kultur- und Lernprogramm hat nicht, wie erhofft, einen ähnlich wichtigen Stellenwert wie die Wettkämpfe.

- Das Sportliche steht für die Teilnehmerinnen und Teilnehmer im Vordergrund; weitere Erfahrungen werden, solang es der teilweise sehr straffe Trainings- und Wettkampfkalender zulässt, gerne mitgenommen.

- Eine langfristige Vermittlung der olympischen Werte durch die YOG scheint nicht möglich zu sein, wohingegen das Interesse an der olympischen Bewegung gesteigert wird.

- Die Mediennutzung und Kommunikation im Rahmen der YOG wurde bisher noch (zu) wenig untersucht und sollte von der Forschung stärker berücksichtigt werden.

- Die neuen Eventformate der YOG können das Programm der Olympischen Spiele nachhaltig beeinflussen, was den wertvollen Innovationscharakter der YOG beweist.

- Tangible und intangible Legacies durch die YOG sind zwar vorhanden, fallen aber wesentlich schwächer aus als bei den Olympischen Spielen dafür betragen die Kosten auch nur einen Bruchteil. 
- Die „Asienlastigkeit“ der YOG im Sommer und die „Europalastigkeit“ im Winter sollten im ständigen co-creation process des IOC berücksichtig werden.

\section{Entstehung und Fokus der Olympischen Jugendspiele}

Im Rahmen der Guatemala-Session 2007 beschloss das Internationale Olympische Komitee (IOC) die Einführung der Olympischen Jugendspiele (YOG). Mit der Implementierung eines globalen Formats wollte IOCPräsident Jacques Rogge, der auch als Initiator des Europäischen Olympischen Jugendfestivals (EYOF) gilt, Akzente in der olympischen Bewegung setzen. Wenngleich das einstimmige Votum seitens der IOC-Funktionäre nach außen hin eine klare Botschaft war, gab es hinter verschlossenen Türen Skepsis (Schnitzer, 2011). Auch aus Sicht der Wissenschaft wurden in Hinblick auf die Ziele der YOG und deren Erreichung Zweifel geäußert (Hanstad et al., 2013; Judge et al., 2009).

Trotz dieser Kritik hielt das IOC am Plan der Durchführung der YOG fest, getrieben vom Ziel, den durchaus nötigen Modernisierungsprozess der olympischen Bewegung voranzutreiben und die Jugend näher an den Sport zu bringen. Um sich Unterstützung zu sichern, machte das IOC den Nationalen Olympischen Komitees (NOCs) und Internationalen Sportfachverbänden Zugeständnisse (z. B. Kostenübernahme bei der Beschickung von Athletinnen und Athleten). Außerdem wurde die Möglichkeit mit neuen Eventformaten zu „experimentieren“ in Aussicht gestellt. Der Kritik, dass die Jugendlichen einer zu frühen Selektion und Leistungsorientierung ausgesetzt wären, sollte mit einem Kultur- und Lernprogramm für die Teilnehmer*innenentgegengewirkt werden.

Im Februar 2009 formulierte das IOC Ziele für die YOG: Diese reichten von der Idee, die besten jungen Athlet"innen der Welt zusammenzubringen, über die Vermittlung von olympischen Werten bis hin zur Diskussion von gesellschaftlichen Herausforderungen. Diese Ziele sollten im Rahmen der YOG in den darauffolgenden zehn Jahren (siehe Tabelle 1) umgesetzt werden. 
Tabelle 1: Überblick über die ersten sechs Ausgaben der Olympischen Jugendspiele

\begin{tabular}{|c|c|c|c|c|c|}
\hline & Datum & Ort & $\begin{array}{l}\text { Athletinnen/ } \\
\text { Athleten }\end{array}$ & NOCs & Events \\
\hline \multirow{3}{*}{$\begin{array}{l}\text { Sommer } \\
\text {-YOG }\end{array}$} & 14.08. - 26.08.2010 & Singapur & 3.524 & 204 & 201 \\
\hline & 16.08. - 28.08.2014 & Nanjing (China) & 3.579 & 203 & 222 \\
\hline & 06.10. - 18.10.2018 & $\begin{array}{l}\text { Buenos Aires } \\
\text { (Argentinien) }\end{array}$ & 4.000 & 206 & 239 \\
\hline \multirow{3}{*}{$\begin{array}{l}\text { Winter- } \\
\text { YOG }\end{array}$} & 13.01. - 22.01.2012 & Innsbruck (Österreich) & 1.022 & 69 & 63 \\
\hline & 12.02. - 21.02.2016 & $\begin{array}{l}\text { Lillehammer } \\
\text { (Norwegen) }\end{array}$ & 1.067 & 71 & 70 \\
\hline & 09.01. - 22.01.2020 & Lausanne (Schweiz) & 1.872 & 79 & 81 \\
\hline
\end{tabular}

Eigene Darstellung nach: IOC (2021)

Überarbeitung der Inhalte der YOG nach der Olympic Agenda 2020

Mit Thomas Bach als Nachfolger von Jacques Rogge wurde der bereits begonnene Reformprozess der olympischen Bewegung intensiviert. Kernstück war und ist die Olympic Agenda 2020 (IOC, 2014). Das Reformwerk hat erhebliche Auswirkungen auf die zukünftige Durchführung der Olympischen Spiele, wie z. B. das Konzept für die Olympischen Winterspiele 2026 in Mailand und Cortina d'Ampezzo zeigt: Laut Plan sollen die Wettkämpfe über fast ganz Norditalien verteilt stattfinden (Schnitzer \& Haizinger, 2019).

Auch die YOG wurden trotz ihres jungen Alters nicht von diesem Reformprozess ausgenommen. Die ersten zehn Jahre der YOG-Geschichte zeigen, dass sich viele ursprünglich geplante Inhalte geändert haben - so wie es auch der vom IOC häufig postulierte co-creation process zur Entwicklung des Eventproduktes YOG vorsieht. Im Rahmen der Agenda 2020 wurden weitere Änderungen beschlossen:

- Die Vision, Mission, Termine, Sportprogramme, Kultur- und Bildungsprogramme, Protokolle, Organisation, Ausrichtung und Finanzierung der YOG wurden neu bewertet.

- Es wurde entschieden, dass die Austragung der YOG auf ein nichtolympisches Jahr gelegt wird, beginnend mit der Verschiebung der 4. YOG von 2022 auf 2023 (IOC, 2014). 
- Eine Schaffung eines „Netzwerks von Jugendsportveranstaltungen“ wurde forciert; Ziel ist ein kohärenter Kalender aus internationalen Veranstaltungen, der seinen Höhepunkt mit den YOG findet.

- Die Einbindung der YOG in den Olympic Channel wurde sichergestellt, um eine höhere Reichweite zu schaffen.

- Es wurde erneut festgehalten, dass die Austragung der YOG allen Städten möglich sein sollte - und zwar durch die Nutzung bestehender und Errichtung temporärer Infrastruktur sowie die Anpassung des Level of Services (IOC, 2017).

- Es wurde festgelegt, dass das Verhältnis aus Teilnehmerinnen und Teilnehmern 50:50 betragen soll; außerdem sollten 70\% der Wettkämpfe im typischen olympischen Format und 30\% in neuen Disziplinen, die sich an den Interessen der Jugendlichen orientieren, stattfinden.

- Es wurde in Aussicht gestellt, dass Athletinnen und Athleten nicht mehr während der ganzen Dauer der YOG vor Ort sein müssen; dies trug dazu bei, dass bei den YOG in Lausanne 2020 ein Teilnehmeranstieg von ca. $70 \%$ zu verzeichnen war (DOSB-Presse, 2017).

Schließlich können die überarbeiteten Ziele der YOG (Abbildung 1) als Zeugnis eines dynamischen Prozesses innerhalb der olympischen Bewegung verstanden werden.

\section{Abbildung 1: Ziele der Olympischen Jugendspiele}

\begin{tabular}{|c|c|}
\hline $\begin{array}{l}\text { Die besten jungen Athlet*innen bei } \\
\text { einem globalen Multisport- } \\
\text { Wettbewerb auf hohem Niveau und } \\
\text { Sportfestival, das kulturelle und } \\
\text { pädagogische Aktivitäten umfasst, } \\
\text { zusammenbringen. }\end{array}$ & $\begin{array}{l}\text { Den kulturellen Austausch und die } \\
\text { olympischen Werte (Nicht- } \\
\text { Diskriminierung, Fair Play, Streben } \\
\text { nach Spitzenleistungen, etc.) } \\
\text { fördern. }\end{array}$ \\
\hline $\begin{array}{l}\text { Mit jungen Menschen zu ihren } \\
\text { Bedingungen über die digitalen } \\
\text { Plattormen der olympischen } \\
\text { Bewegung (einschließlich } \\
\text { Olympic Channel und IOC- } \\
\text { Website) in Kontakt treten. }\end{array}$ & $\begin{array}{l}\text { Ein Katalysator für Innovation und } \\
\text { Inkubator für neue Konzepte sein: } \\
\text { - neue Sportarten und } \\
\text { Wettbewerbsformate testen und } \\
\text { validieren, } \\
\text { - Bildungsinitiativen für junge } \\
\text { Menschen schaffen, } \\
\text { - Technologien, die sich auf } \\
\text { nachhaltigere Veranstaltungsmodelle } \\
\text { konzentrieren, forcieren, } \\
\text { - Programme, die digital geteilt werden } \\
\text { können, schaffen. }\end{array}$ \\
\hline
\end{tabular}

(IOC, 2019) 
Erkenntnisse aus wissenschaftlichen Studien zu den YOG

Ziel dieses Beitrages ist es, ein Resümee der ersten zehn Jahre Olympische Jugendspiele zu ziehen. Wenngleich die vorliegende Arbeit nicht als systematische Literaturanalyse verstanden werden darf, soll sie einen guten Überblick geben und somit aufzeigen, zu welchen Themen rund um die YOG geforscht wurde und welche Schlüsse nach zehn Jahren Olympische Jugendspiele und Forschung zu den YOG gezogen werden können. Insgesamt wurden 285 Arbeiten, die den Begriff Youth Olympic Games im Titel beinhalten, analysiert (Tabelle 2). Die Ergebnisse werden in Hinblick auf die zuvor genannten Ziele des IOC zusammengefasst.

Tabelle 2: Suchergebnisse nach dem Begriff, Youth Olympic Games“ auf „Web of science"

\begin{tabular}{|c|c|}
\hline Zeitraum & Treffer (285) \\
\hline Bis 2005 & 10 \\
\hline 2006 bis 2010 & 29 \\
\hline 2011 bis 2015 & 101 \\
\hline Seit 2015 & 145 \\
\hline
\end{tabular}

Analyse Ziel 1 - Zusammenführung der besten Atbletinnen und Atbleten in einem Sportfestival

Hinsichtlich des Ziels, die besten jungen Athletinnen und Athleten bei einem globalen Multisport-Wettbewerb mitsamt kulturellen und pädagogischen Aktivitäten zusammenzubringen, herrscht in der Wissenschaft Einigkeit: Dem IOC und den Organisatoren ist es nur schwer gelungen, die jugendliche Bevölkerung der Welt für Sport und körperliche Aktivität zu begeistern. Ein wesentlicher Grund ist, dass die Teilnehmerzahl sehr limitiert ist (insbesondere bei den Winter-YOG mit 1.000 Athletinnen und Athleten) und es enorme Ressourcen bräuchte, um auch Jugendliche zu erreichen, die nicht an den Spielen teilnehmen (Schnitzer, 2011).

In der bisherigen Analyse der YOG hat das Kultur- und Lernprogramm (vorher Culture and Education Programme, später Learn and Share Programme. (IOC, 2009)) einen großen Stellenwert eingenommen. Am Beispiel der YOG in Singapur zeigte Krieger (2012) die Diskrepanz in der Wahrnehmung des Erfolgs des Kulturprogrammes zwischen Athlet*innen 
und Organisator*innen auf; hingegen wurde der informelle Austausch der Jugendlichen im olympischen Dorf sehr positiv wahrgenommen. In Bezug auf die YOG 2012 in Innsbruck nahmen Schnitzer et al. (2014), Kristiansen (2015), Peters und Schnitzer (2015) sowie Tanabe et al. (2015) umfassende quantitative und qualitative Analysen zur Wahrnehmung des Kulturprogrammes vor. Fazit: Das Kulturprogramm kam bei allen jungen Athlet*innen, die daran teilgenommen haben, sehr gut an, allerdings verwehrte der sehr enge Trainings- und Wettkampfplan rund der Hälfte der Jugendlichen das Mitmachen, was zu Kritik führte. Zudem wurde festgestellt, dass die Athlet*innen zwar wettkampferfahren sind, aber die kulturelle, soziale und internationale Dimension der YOG ganz offensichtlich eine herausragende Erinnerung hinterlässt. Dennoch zeigte die zusammenfassende Analyse eindeutig, dass das Hauptziel der YOG-Athlet*innen die Teilnahme an den sportlichen Wettkämpfen ist. Um ihr Interesse am Kulturprogramm zu stärken, müssten diese Aktivitäten in kurzer Entfernung zu den Wettkampfstätten und der Unterkunft angeboten werden. Außerdem besteht die Notwendigkeit, diese Zusatzprogramme sorgfältiger zu planen und das Interesse der Trainer*innen sowie der Chefs de Mission diesbezüglich zu erhöhen.

Diese Ergebnisse, die sich hauptsächlich auf die Innsbruck-Ausgabe 2012 beziehen, konnten mit einer Studie zu den YOG 2016 in Lillehammer (Krieger \& Kristiansen, 2016) eins-zu-eins bestätigt werden. Das heißt, dass die YOG bei der Erreichung der Bildungsziele der Olympischen Bewegung nur begrenzt Erfolg hatten. Eine retrospektive Analyse der Teilnehmer*innen von Singapur (fünf Jahre nach Teilnahme) und Innsbruck (drei Jahre nach Teilnahme) zeigte, dass die YOG für die Sportler"innen das Highlight ihrer sportlichen Karriere war (Schnitzer et al., 2017). Die Mehrheit von ihnen gab an, dass die YOG die wichtigste Veranstaltung in ihrem Wettkampfkalender gewesen sei. Nach eigenen Angaben wurden $77 \%$ der Befragten durch die YOG animiert, mit dem Leistungssport weiterzumachen. Diese Ergebnisse konnten in der Studie von Kristiansen et al. (2018) bestätigt werden. Sie stellten außerdem fest, dass die Drop-Out-Raten ehemaliger YOG-Athlet"innen nicht im Zusammenhang mit dem Gewinnen bzw. Nicht-Gewinnen einer Medaille stehen. Zudem zeigte sich, dass der zeremonielle Charakter der YOG mit Eröffnungs-, Schluss- und Medaillenfeier für die Athlet"innen äußerst einprägsam war. Zudem stellte die Kommunikation zwischen den nationalen Delegationen, auch außerhalb der eigenen Sportart, eine besondere Erfahrung dar (Kristiansen et al, 2018; Peters \& Schnitzer, 2015).

Was sich für die Ausgaben von 2010 bis 2016 zeigte, scheint auch für die YOG 2018 in Buenos Aires zu gelten: MacIntosh et al. (2019) konnten 
nicht nur frühere Ergebnisse bestätigen, sondern auch aufzeigen, dass die jungen Athlet"innen ihre YOG-Teilnahme als vorteilhaft empfanden - insbesondere das Sammeln von Wettkampferfahrungen sowie die geplanten und ungeplanten sozialen Interaktionen während der Veranstaltung. Durch ihre Erfahrungen bei den YOG bildeten die Jugendlichen ihr eigenes Selbstverständnis innerhalb der vom IOC geschaffenen und propagierten Olympischen Bewegung aus. Dies ging einher mit der Entwicklung ihrer Identität: Sie lernten, sich über Grenzen hinweg von Mitgliedern eines Nationalteams zu internationalen, multisportlichen YOG-Athlet*innen zu entwickeln.

Für die Jugendlichen liefern die YOG einen ersten Überblick über das olympische System sowie die Erwartungen und Privilegien, die mit dem leistungsstärksten Multisportsystem der Welt verbunden sind. Schließlich zeigten Nordhagen und Fauske (2019) die Bildungspotenziale (z.B. Umgang mit Olympischen Werten, Kennenlernen von Sport und Bewegung als Teil eines gesunden Lebensstils) der YOG auf, monierten zugleich aber die Schwächen in der Durchführung und Gestaltung der Inhalte der Lernprogramme im Rahmen der Olympischen Jugendspiele.

\section{Analyse Ziel 2 - Förderung olympischer Werte}

In Bezug auf das zweite Ziel - die Förderung des kulturellen Austauschs und der olympischen Werte, einschließlich Nicht-Diskriminierung, Fair Play und Streben nach Spitzenleistungen - kann zum Teil auf die zuvor erwähnten Studienergebnisse zurückgegriffen werden. Wie die zusammenfassende Literaturanalyse von De Souza und Tavares (2020) zeigte, kann die Teilnahme an YOG bei jungen Sportler*innen eine Reihe von wertebasierten Lernerfahrungen fördern. Allerdings scheinen sich solche Lernerfahrungen viel mehr aus informellen Treffen und soziokulturellen Interaktionen zwischen den Jugendlichen zu ergeben als durch organisierte Programme. De Souza und Tavares (2020) resümierten, dass der Erfolg dieses neuen Events nicht in der Nachahmung der Olympischen Spiele mit dem Ziel, Jugendliche auf die „Erwachsenen-Ausgabe“ vorzubereiten, liegt, sondern in der Fähigkeit, die Triade "Sport-Wettkampf-Bildung“ besser auszubalancieren.

Hinsichtlich der Förderung (olympischer) Werte wurde in der Literatur vornehmlich analysiert, wie Jugendliche, die nicht als Teilnehmer*innen am Event beteiligt waren, die YOG wahrgenommen haben. So stellten sich Prüschenk und Kurscheidt (2017) die Frage, ob es den YOG gelingt, olympische Wertvorstellungen zu verändern. Die Ergebnisse zeigten, 
dass Jugendliche der Vermittlungsfunktion der Olympischen Idee eher skeptisch gegenüberstehen. Jugendliche schätzen vielmehr die Sportkultur der Olympischen Spiele, während ältere Befragte auch die wirtschaftlichen Notwendigkeiten der Ausrichtung der Olympischen Spiele in der Meinungsbildung berücksichtigen. Die Ergebnisse der Arbeit stützen die Ansicht, dass die YOG das Potenzial haben, junge Menschen zu erreichen, und dass diese für olympische Werte empfänglich sind. Somit scheinen die YOG geeignet, das Brückenkapital des Olympismus zu fördern, vor allem bei Jugendlichen.

Schnitzer et al. (2018) untersuchten in ihrer empirischen Arbeit die Beteiligung der lokalen Jugend an den YOG (über Schulprogramme, Medien oder Vor-Ort-Besuche der YOG) und maßen den Einfluss auf die Wahrnehmung olympischer Werte. Sie kamen zum Schluss, dass die YOG zwar in der Lage sind, das allgemeine Interesse an der Olympischen Bewegung zu wecken, jedoch die Wahrnehmung der olympischen Werte nicht signifikant beeinflussen. Vielmehr hängt die Wahrnehmung der olympischen Werte vom soziodemographischen Hintergrund, dem grundsätzlichen Interesse an Sportereignissen, dem sozialen Kapital und dem Ausmaß, in dem die Jugendlichen die Veranstaltung in den verschiedenen Medien verfolgen, ab. Folglich dürfen Interventionsprogramme (z. B. Schulprogramme) zur Förderung von (olympischen) Werten und Idealen keine einzelnen, obligatorischen und isolierten Aktivitäten sein.

\section{Analyse Ziel 3 - Nutzung digitaler Plattformen als Brückenschlag zur olympischen Bewegung}

Bisherige Arbeiten zum dritten Ziel - mit jungen Menschen zu ihren Bedingungen über die digitalen Plattformen der Olympischen Bewegung, einschließlich Olympic Channel und IOC-Webseite, in Kontakt treten - zielten die auf die öffentliche Wahrnehmung und Nutzung sozialer Kommunikationsplattformen im Rahmen der YOG ab. Judge et al. (2013) zeigten auf, wie das IOC hinsichtlich Social-Media-Kampagnen dazugelernt hat. Aufgrund ihres interaktiven Contents wurden die YOG 2012 als positives Beispiel genannt. Judge et al. (2013) empfehlen, die Marketingmaßnahmen der YOG auf die Millennials (Generation der Jahrtausendwende) auszurichten, um Glaubwürdigkeit zu schaffen und die Botschaften der YOG auf die Kommunikationsebene dieser Zielgruppe zu setzen, ohne unerwünschte Überkommerzialisierung zu propagieren. An diese Thematik anknüpfend analysierten Naraine und Parent (2017) die Kommunikation der YOG-Organisationskomitees über das Medium 
Twitter. Sie kamen zum Schluss, dass die Tweets eher werbebezogene Inhalte als informations- und berichtsbezogene Inhalte lieferten, dass sich die Themen bzw. die Struktur im Laufe der Zeit von 2010 bis 2016 wesentlich verbessert hat, und dass zunehmend stärker auf die Zielgruppe der Jugendlichen eingegangen wurde.

\section{Analyse Ziel 4 - Die YOG als Katalysator für Innovationen}

Aus Sicht des ICO sollen die YOG als Katalysator für Innovation und Inkubator für neue Konzepte fungieren. Dazu zählen z. B. das Testen neuer Sportarten und Wettbewerbsformate, Bildungsinitiativen für junge Menschen oder Technologien, die sich auf nachhaltigere Veranstaltungsmodelle konzentrieren. Schnitzer (2012) und Schnitzer et al. (2014) analysierten die im Rahmen der YOG 2012 eingeführten neuen Eventformate aus Sicht des Publikums und der Medienvertreter. Beide Stakeholdergruppen zeigten ein ausgeprägtes Interesse an den neuen Wettbewerben und stuften das sportliche Leistungsniveau in diesen als sehr positiv ein. Bezogen auf den Unterhaltungswert, die zukünftige Entwicklung, den Eustress und die Regeln nahmen die Zuschauenden die einzelnen Wettbewerbe unterschiedlich wahr. Im Einklang mit Torres (2020) zeigten die Studien, dass die Olympischen Jugendspiele innerhalb der Olympischen Bewegung ein wichtiges Testfeld für Innovationen darstellen: Beispielsweise wurden Wettbewerbe, die in Innsbruck im Rahmen der YOG durchgeführt wurden, bei den Winterspielen in Sotschi 2014 erstmals ins olympischen Programm aufgenommen.

\section{Erkenntnisse aus Legacy-Studien zu den YOG}

Mit den YOG als Fallbeispiel ist es unter anderem gelungen, wissenschaftliche Analysen zum Vermächtnis - den Legacies - der Jugendspiele durchzuführen. Diese haben deshalb innovativen Charakter, weil sie zum Teil mehrere Jahre nach den YOG vorgenommen wurden. So konnten Seidl et al. (2019) feststellen, dass Innsbrucker bzw. Tiroler Jugendliche überwiegend positiven Assoziationen zu und eine positive Meinung über die YOG hatten. Obwohl die Mehrheit der Jugendlichen die Ausrichtung der YOG 2012 in Innsbruck als eine gute Sache empfand, sahen nur sehr wenige die Veranstaltung als entscheidenden Punkt in ihrem Leben. Folglich waren die YOG 2012 nicht „stark“ genug, um herausragende 
Vermächtnisse zu schaffen, verursachten aber auch keinen „Schaden“ im Sinne von negativen Vermächtnissen. Schließlich zeigten Schnitzer et al. (2019) auf, dass die Einbindung lokaler Jugendlicher in die YOG und die Erfahrungen, die sie dadurch gewinnen, einen signifikanten Einfluss auf die Unterstützung zukünftiger Olympiabewerbungen von Seiten der Bevölkerung haben.

In einem weiteren Beitrag zu Legacies widmeten sich Sand et al. (2016) den Volunteers der YOG 2016. Diese äußerten sich sehr positiv über ihre allgemeinen Erfahrungen als Freiwillige und darüber, wie diese Erfahrungen ihre Bereitschaft für zukünftige Freiwilligenarbeit beeinflusst haben. Übergeordnete Sportinstitutionen könnten die Einrichtung von Freiwilligenvereinigungen in Erwägung ziehen, die speziell daran arbeiten, die Freiwilligentätigkeit mit den Motiven der heutigen EventVolunteers in Einklang zu bringen, um die Bindung zu stärken. Dies wurde beispielsweise nach den YOG 2012 in Innsbruck umgesetzt. Das daraus entstandene Volunteerteam blieb über mehrere Jahre bis heute bestehen.

\section{Fazit: Zehn Jahre Olympische Jugendspiele}

Abschließend stellen sich nun zwei Fragen: Erstens, haben die YOG „etwas gebracht" - und wenn ja, wem? Und zweitens, kann diese sehr idealistisch ausgerichtete Initiative als ein kurz andauerndes „Facelifting“ der olympischen Bewegung angesehen werden, oder haben die YOG tatsächlich auch eine „Verjüngungskur" bewirkt? Auf Basis der vorliegenden empirischen Evidenz kann folgendes festgehalten werden:

- Aufgrund der Einschränkung der Teilnehmerzahl ist das Ziel, die besten Sportler*innen in den jeweiligen Altersgruppen im Rahmen der YOG zusammenzubringen, rein rechnerisch sehr schwierig zu erreichen; wenngleich das Wettkampfniveau als sehr hoch eingestuft werden kann. Für die Teilnehmer"innen stellen die YOG eine unvergessliche Erfahrung dar und werden als Karrierehighlight angesehen, auch über den Zeitraum der YOG hinweg. Zudem konnte festgestellt werden, dass die Teilnahme an den YOG kein Prädiktor für den weiteren Karriereverlauf ist.

- Die Vorstellung, dass Athlet*innen dem Kultur- und Lernprogramm eine ähnlich wichtige Rolle wie dem Wettkampf zumessen, musste bereits nach den ersten Ausgaben der YOG in der Kategorie "frommer Wunsch“ abgelegt werden. Der sportliche Wettkampf steht für die 
Teilnehmenden und ihrem Umfeld ganz klar im Vordergrund. Wenn es darüber hinaus die Möglichkeiten gibt, weitere Erfahrungen mitzunehmen, wird dieses Angebot gerne angenommen, solange der Trainings- und Wettkampfalltag nicht darunter leidet.

- Ob die olympischen Werte im Rahmen der YOG gezielt an junge Athlet*innen, aber auch die Jugendlichen der Gastgeberstadt weitergegeben werden, muss bezweifelt werden. Die empirischen Befunde zeigten ganz klar, dass die YOG bei Jugendlichen zwar das Interesse an der Olympischen Bewegung wecken, die Vertretung von (olympischen) Werten jedoch stärker durch die Sozialisierung geprägt ist.

- Was die Mediennutzung und Kommunikation im Rahmen der YOG betrifft, ist der empirische Kenntnisstand eher dünn. Hier sollte die Forschung ansetzen, denn die Frage, wie Jugendlichen die Botschaften der YOG bestmöglich vermittelt werden können, wird auch in Zukunft eine wesentliche Rolle spielen. Studien wie jene von Schnitzer et al. (2018) haben gezeigt, dass die Medien in der Wahrnehmung der Vermächtnisse der YOG eine wesentliche Rolle spielen.

- Schließlich wurde ersichtlich, dass die YOG mit neuen Eventformaten (z. B. gemischtgeschlechtliche Staffeln bzw. Team-Events, Staffeln bzw. Team-Events mit Sportler*innen aus unterschiedlichen Nationen, eine Kombination aus beidem oder neuen Wettbewerben) das Programm der Olympischen Spiele nachhaltig beeinflusst haben. Dies untermauert den Innovationscharakter der YOG.

Einmal mehr zeigten die YOG, unter anderem aus Mangel an neu zu entwickelnder Infrastruktur, dass Sportgroßveranstaltungen nicht nur nach tangiblen, sondern auch nach intangiblen Legacies beurteilt werden müssen. Klarerweise sind diese bei weitem nicht so bedeutend, wie jene der Olympischen Spiele - im positiven wie auch im negativen Sinne. Umgekehrt sind nach Schnitzer und Chappelet (2014) die Kosten der Durchführung der YOG (inkl. Infrastrukturmaßen, die meist nicht als olympiabedingte Kosten gelten) nur ein Bruchteil jener der Olympischen Spiele. Es scheint also kein Zufall, dass die Olympic Agenda 2020 mit den YOG im Rücken da oder dort etwas mutiger ausgefallen ist. Dennoch gibt es einige „YOG-Baustellen“, die in den nächsten Jahren erledigt werden müssen (z.B. Sichtbarkeit der YOG in der breiten Bevölkerung, Finanzierung der YOG).

Ungeachtet der COVID-19-Pandemie und der Verschiebung der ersten afrikanischen Spiele im Senegal in das Jahr 2026 ist die Frage künftiger Austragungsstädte zu lösen. Die „Asienlastigkeit“ der YOG im Sommer und die „Europalastigkeit“ der YOG im Winter wird Teil des ständigen $c o^{-}$ 
creation process sein. Dieser wiederum brachte lokalen Organisator*innen der YOG von Beginn an in eine bedeutende Rolle bei der Entwicklung der Olympischen Jugendspiele. Die wissenschaftliche Begleitung der YOG mithilfe des Olympic Study Center, der nationalen Olympischen Akademien oder Initiativen wie dem Youth Olympic Games Laboratory for Youth and Innovation (YOGINN) in Innsbruck 2012 (Schnitzer, 2012) soll auch in Zukunft dazu beitragen, das Phänomen der YOG evidenzbasiert zu untersuchen.

\section{Literatur:}

De Souza, A. L., \& Tavares, O. (2020). The educational experience of athletes in Youth Olympic Games: A systematic review. Movimento, 26, e26039. https://doi. org/10.22456/1982-8918.97317

DOSB-Presse (2017). Die Entscheidungen der 131. IOC-Session. Abgerufen unter https://www.teamdeutschland.de/news/details/die-entscheidungen-der -131-ioc-session

Hanstad, D. V., Parent, M. M., \& Kristiansen, E. (2013). The Youth Olympic Games: The best of the Olympics or a poor copy? European Sport Management Quarterly, 13(3), 315-338. https://doi.org/10.1080/16184742.2013.782559

IOC (2009). Factsheet Youth Olympic Games Update - February 2009. Abgerufen unter https://stillmed.olympic.org/Documents/Reports/EN/en_report_1423.pdf

IOC (2014). Olympic Agenda $202020+20$ Recommendations. Abgerufen unter https://stillmed.olympic.org/Documents/Olympic_Agenda_2020/ Olympic_Agenda_2020-20-20_Recommendations-ENG.pdf

IOC (2017). IOC Reveals the Future of the Youth Olympic Games. Abgerufen unter https://www.olympic.org/news/ioc-reveals-the-future-of-the-youth-olympic -games

IOC (2019). Factsheet the YOG - Vision, birth and principles. Abgerufen unter https:/ /stillmedab.olympic.org/media/Document\%20Library/OlympicOrg/FactsheetsReference-Documents/Games/YOG/Factsheet-The-YOG-Vision-Birth-and-Princi ples.pdf

IOC (2021). Olympic Games. Youth Olympic Games. Abgerufen unter https://www. olympic.org/singapore-2010; https://www.olympic.org/innsbruck-2012; https:/ /www.olympic.org/nanjing-2014; https://www.olympic.org/lillehammer-2016; https://www.olympic.org/buenos-aires-2018; https://www.olympic.org/lausanne $-2020$

Judge, L. W., Bellar, D., Petersen, J., Wanless, E., Surber, K., Gilreath, E., \& Simon, S. (2013). Olympic Games Evolution: An Analysis of Personal and Public Awareness of the Inaugural Youth Olympic Games. In A. R. Hofmann, \& M. Krüger (Hrsg.), Olympia als Bildungsidee, Bildung und Sport (S. 237-248). Springer Verlag. https://doi.org/10.1007/978-3-531-19552-0_12 
Judge, L. W., Petersen, J., \& Lydum, M. (2009). The best kept secret in sports: The 2010 Youth Olympic Games. International Review for the Sociology of Sport, 44(2-3), 173-191.

Krieger, J. (2012). Fastest, highest, youngest? Analysing the athlete's experience of the Singapore Youth Olympic Games. International Review for the Sociology of Sport, 706-719. https://doi.org/10.1177/1012690212451875

Krieger, J., \& Kristiansen, E. (2016). Ideology or reality? The awareness of educational aims and activities amongst German and Norwegian participants of the first summer and winter Youth Olympic Games. Sport in Society, 19(10), 15031517. https://doi.org/10.1080/17430437.2015.1133604

Kristiansen, E. (2015). Competing for culture: Young Olympians' narratives from the first winter Youth Olympic Games. International Journal of Sport and Exercise Psychology, 13(1), 29-42. https://doi.org/10.1080/1612197X.2012.756259

Kristiansen, E., MacIntosh, E. W., Parent, M. M., \& Houlihan, B. (2018). The Youth Olympic Games: A facilitator or barrier of the high-performance sport development pathway? European Sport Management Quarterly, 18(1), 73-92. https://doi.org/10.1080/16184742.2017.1383499

MacIntosh, E. W., Parent, M. M., \& Culver, D. (2019). Understanding Young Athletes' Learning at the Youth Olympic Games: A Sport Development Perspective. Journal of Global Sport Management. https://doi.org/ 10.1080/24704067.2018.1561206

Naraine M. L., \& Parent, M.M. (2017). The evolution of Twitter communication by Youth Olympic Games organising committees. International Journal of Sport Management and Marketing, 17, 403-425.

Nordhagen, S. E., \& Fauske, H. (2019). The Youth Olympic Games as an arena for Olympic education: An evaluation of the school program, "Dream Day". AUC Kinanthropologica, 54(2), 79-95.

Peters, M., \& Schnitzer, M. (2015). Athletes' Expectations, Experiences, and Legacies of the Winter Youth Olympic Games Innsbruck 2012. Journal of Convention \& Event Tourism, 16(2), 116-144. https://doi.org/ 10.1080/15470148.2015.1018656

Prüschenk, N., \& Kurscheidt, M. (2017). Do the Youth Olympic Games have the potential to shift perceptions of Olympism? Evidence from young people's views on Olympic values. Int. J. Sport Management and Marketing, 17(4/5/6), 351-380.

Sand, T. S., Strittmatter, A.-M., \& Hanstad, D. V. (2016). 2016 Winter Youth Olympic Games: planning for a volunteer legacy. International Journal of Sport Management and Marketing, 17, 242-260.

Schnitzer, M. (2011). Challenge vs internationalism intercultural meeting at the winter Youth Olympic Games 2012. In H. Preuss, \& K. Liese (Hrsg.), Internationalism in the Olympic Movement. (S.113-125). Wiesbaden: VS Verlag für Sozialwissenschaften. https://doi.org/10.1007/978-3-531-92891-3_10

Schnitzer, M. (2012). Evaluation of the innovative elements of the WYOG 2012 Sports Programme with particular emphasis on the media and spectators. Final Report for the 2012 Post Graduate IOC-Olympic Studies Centre Grant. 
Schnitzer, M., Bork, M., Eder, T., Zobl, N., \& Schlemmer, P. (2017). Intangible Effekte von Sportgroßveranstaltungen aus Sicht von Athlet/innen am Beispiel der Olympischen Jugendspiele 2010 und 2012. German Journal of Exercise and Sport Research, 47, 301-304.

Schnitzer, M., \& Chappelet, J.-L. (2014). Bidding for and financing the Youth Olympic Games. In D. V. Hanstad, M. M. Parent, \& B. Houlihan (Hrsg.), The Youth Olympic Games (S. 53-74). London: Routledge, Taylor \& Francis.

Schnitzer, M., \& Haizinger, L. (2019). Does the Olympic Agenda 2020 have the power to create a new Olympic heritage? An analysis for the 2026 Winter Olympic Games Bid. Sustainability, 11(2), 442. https://doi.org/10.3390/ su11020442

Schnitzer, M., Peters, M., Scheiber, S., \& Pocecco, E. (2014). Perception of the Culture and Education Programme of the Youth Olympic Games by the participating athletes: A case study for Innsbruck 2012. The International Journal of the History of Sport, 31(9), 1178-1193. https://doi.org/10.1080/09523367.2014.909810

Schnitzer, M., Walde, J., Scheiber, S., Nagiller, R., \& Tappeiner, G. (2018). Do the Youth Olympic Games promote Olympism? Analysing a mission (im)possible form a local youth perspective. European Journal of Sport Science, 18(5), 722-730.

Schnitzer, M., Walde, J., Scheiber, S., Nagiller, R., \& Tappeiner, G. (2019). Does the young residents' experience with the Youth Olympic Games influence the support for staging the Olympic Games? Tourism Management Perspectives, 20, 220-231.

Seidl, M., Nagiller, R., Lang, A., Scheiber, S., \& Schnitzer, M. (2019). Youth Olympic Games (YOG) 2012-Mission accomplished? A retrospective analysis of intangible legacies and the fulfillment of the YOG's goals. Journal of Global Sport Management. https://doi.org/10.1080/24704067.2019.1642122

Tanabe, Y., Asakawa, S., Arakida, Y., Kono, I., \& Akama, T. (2015). Characteristics of the Japanese national team of the first Youth Olympic Games. Journal of Sports Medicine \& Doping Studies, 5(2).

Torres, C. R. (2010). The Youth Olympic Games, their programs, and Olympism. Kinesiology, Sport Studies and Physical Education Presentations and Papers, 3. 